Chapter 7

\title{
Chromatin Remodeling in \\ DNA Damage Response and Human Aging
}

\author{
Lili Gong, Edward Wang and Shiaw-Yih Lin \\ Additional information is available at the end of the chapter \\ http://dx.doi.org/10.5772/55272
}

\section{Introduction}

Chromatin consists of the DNA and all proteins involved in organizing and regulating DNA structure. The building block of chromatin is the nucleosome, which is composed of 146 base pairs of DNA and a core histone octamer. The core histone oactomer is composed of two heterodimers of histone $\mathrm{H} 2 \mathrm{~A}$ and histone $\mathrm{H} 2 \mathrm{~B}$ and a tetramer of histone $\mathrm{H} 3$ and histone $\mathrm{H} 4$ [1]. The overall chromatin structure is very dynamic in response to diverse biological events. Regulation of chromatin structure is achieved by two major mechanisms. The first is posttranslational modification (PTM) of histones and other chromatin proteins via phosphorylation, methylation, acetylation, ubiquitination and sumoylation $[2,3]$. The second is through ATP-dependent nucleosome structure alteration. Cooperation between histone PTMs and chromatin remodelers allows chromatin remodeling to regulate diverse biological events including transcription, chromosome segregation, DNA replication, and DNA repair. In this chapter, we summarize how chromatin structure is regulated during DNA damage response (DDR), focusing particularly on three PTMs: phosphorylation, Poly(ADP-ribosyl)ation (PARylation) and sumoylation. We discuss the DDR in a highly compacted chromatic structure, heterochromatin, as well as the interplay between chromatin remodeling, DNA damage and human aging.

\section{DNA damage response}

\subsection{Sources leading to DNA damage}

In order to maintain DNA fidelity, cells must overcome multiple challenges that threaten genome stability. Cues cause DNA damages can be divided into spontaneous and environ- 
ment-induced. Spontaneous DNA damages are usually caused by intracellular metabolism stress, or formed during genetically programmed processes such as $\mathrm{V}$ (variable), D (diversity), and $\mathrm{J}$ (joining) ( $\mathrm{V}(\mathrm{D}) \mathrm{J}$ ) recombination in developing vertebrate lymphocytes or meiotic recombination in germ cells $[4,5]$. The major types of damage include aberrant conformations of DNA, chemical instability of DNA, free radicals of oxygen, endogenous mutagens and errors in DNA replications [6]. Environmental DNA damages generally refer to exposure of cells to various genotoxic agents. These agents contain both physical factors, such as ultraviolet (UV), visible light and ionizing radiation; as well as chemical factors, such as alkylating agents, benzopyrene, aflatoxins and cis-Platinum. These DNA damages can lead to single base mutation or more deleterious chromosomal lesion.

\subsection{DDR pathway}

To maintain genome stability, cells have developed a global signaling network, known as the DNA damage response (DDR), to sense different types of genotoxic stress, to modulate cell cycle transitions and transcriptional process, and to stimulate DNA repair. Mechanistically, proteins involved in the DDR signaling network can be grouped into three major classes: 1) Sensors, acting at the upstream of DDR by recognizing the DNA damage and initiating DDR; 2) Transducers, proteins that pass and amplify DNA damage signals to downstream effectors. Notably, among diverse transducers, ATM (Ataxia Telangiectasia Mutated) and ATR (ATM and Rad3 Related) are central to the entire DDR; 3) Effector, proteins determine the physiological outcome of DNA damage response. Depending on the context of DNA damage, effectors can regulate cell cycle, transcription or cell apoptosis. Nevertheless, we need to point out that although DDR is often referred to as a signaling pathway, it is more accurately described as a network of interacting pathways that coordinate the damage response.

\subsection{DNA damage repair}

The various types of DNA damage include aberrant base or nucleotide modifications, single strand DNA (ssDNA) breaks, and chromosomal lesions caused by double strand breaks (DSBs). Among these, DSBs are regarded as the most cytotoxic. If left unrepaired, DSBs will affect genome integrity by causing mutations, chromosome deletions or translocations, because there is no intact complimentary template to repair the damaged strand. In this chapter, we will use DSBs as a model lesion.

DSBs can be repaired by two principle mechanisms, the non-homologous end joining (NHEJ) and homologous recombination (HR) [7]. These two pathways differ in their functional enzymes, the repair efficiency and also the cell cycle phases where they are active (Figure 1).

Molecular Basis of NHEJ. In the process of NHEJ, DSBs are repaired by direct ligation of the exposed ends regardless of their DNA sequences. Enzymes involved specifically in NHEJ capture both ends of the broken DNA molecule, bringing them together to form a DNA-protein complex to repair the break. Therefore NHEJ is a very efficient but error-prone way to repair damaged DNA, and it occurs in all phases of the cell cycle [8]. In NHEJ pathway, the Ku70/80 heterodimer initiates NHEJ by binding to both ends of the broken DNA molecule, which 
creates a scaffold for the assembly of other NHEJ enzymes. After association of Ku70/80, the DNA-dependent protein kinase catalytic subunit (DNA-PKcs) is recruited to the DSB, forming a synaptic complex that brings the broken DNA ends together. Once the DNA ends have been captured and tethered, non-ligatable DNA termini must be processed (single strand fill in) or removed by nucleases and polymerases. Lastly, the processed DNA ends are joint by ligase IV/XRCC4 complex [9]. It was demonstrated that in higher eukaryotes, and especially in mammals, NHEJ is the preferred pathway for DNA DSB repair [10].

Molecular Basis of HR. HR is an error-free, template-dependent strategy to repair DNA DSBs. It occurs during the $S$ and G2 phases, when the sister chromatids are more easily available [11]. The key reactions in HR are homology search and DNA strand invasion, which are catalyzed by the RecA homolog Rad51 [12]. Principally, HR can be divided into three steps: 1) Presynapsis. During this process, DSB ends are recognized and processed to a single-stranded tail with a 3 '-OH ending. This ssDNA is then bound by the eukaryotic ssDNA binding protein RPA (replication protein A). 2) Synapsis. With aid of cofactors, such as Rad 52 and Rad 55-57 complexes [13, 14], Rad51 binds to RPA-coated ssDNA, forming Rad51-DNA filament. After this, DNA strand invasion by the Rad51-ssDNA filament generates a D-loop intermediate. 3) Postsynapsis. In DSBs repair, both ends of the DSB are engaged, leading to double Holliday junction formation. Finally, following the actions of polymerases, nucleases and helicases, DNA ligation and substrate resolution occur.

How cells choose between NHEJ or HR to repair the DSBs is unclear. As mentioned above, $\mathrm{HR}$ is initiated by ssDNA resection and requires sequence homology, but NHEJ requires neither resection at initiation nor a homologous template. Thus, resection appears to be a pivotal step in DSB repair initiation that determines whether HR or NHEJ occurs. However, an alternative end-joining (A-EJ) pathway, which is also initiated by ssDNA resection but does not require a homologous partner, was recently identified. It is proposed that the presence of ssDNA resection will determine the selection among canonical NHEJ, A-EJ or HR; the size of the resection, which is associated with the cell cycle phase, will then direct the DSB repair to either HR or A-EJ [15].

\section{Chromatin remodeling during DNA damage}

In 1998, functions of phosphorylation at the Serine139 residue of a histone variant, H2AX, were first discovered in DDR [16]. Since then, extensive studies regarding how chromatin alters its structures in response of DNA damage were made. It is now recognized that small DNA lesion can lead to global chromatin remodeling, with changes including histone modifications, nucleosome positioning and higher-order folding of the chromatin fiber. Furthermore, if the DDR-induced chromatin remodeling is not properly restored, then the epigenetic changes can be heritable and contribute to terminal cell fate, such as transformation, cell senescence and cell apoptosis $[17,18]$. In this part, we will discuss recent progress made about chromatin structures regulations in the detecting and repairing process of DNA damage. Specifically, we will focus on H2AX phosphorylation regulation, Poly (ADPribosyl)ation, and sumoylation in DDR. 


\subsection{H2AX phosphorylation}

One of the key events that initiates DDR is phosphorylation at the Ser139 of histone H2AX, a chromatin-bound histone variant compromising up to $25 \%$ of the H2A [16]. This phosphorylation process is catalyzed by the master regulator of DDR, ATM and ATR. Phosphorylation of $\mathrm{H} 2 \mathrm{AX}$ at Ser139 is very rapid and this phosphorylated $\mathrm{H} 2 \mathrm{AX}(\gamma \mathrm{H} 2 \mathrm{AX})$ serves as a platform, directly recruiting Mdc1 (mediator of DNA-damage checkpoint 1), and additional factors such as 53BP1, RNF8, and the BRCA1A complex to affected sites [19].

Although $\gamma \mathrm{H} 2 \mathrm{AX}$ is a well-recognized marker for DNA damage, its precise role in chromatin remodeling is only just becoming clear. It was recently found that phosphorylation at a tyrosine site, Tyr142, plays a pivotal role in regulating H2AX functions in DDR [20]. Basal phosphorylation of H2AX at Tyr142 was carried out by WSTF (Williams syndrome transcription factor), a component of the WICH ATP-dependent chromatin remodeling complex [20]. At the early stage of DDR $(<1 \mathrm{hr})$, inhibition of phosphorylation at Tyr142 by knocking down WICH did not affect $\gamma \mathrm{H} 2 \mathrm{AX}$ foci formation, but during the later recovery stages, $\gamma \mathrm{H} 2 \mathrm{AX}$ foci was greatly reduced[20]. So it seems that in the absence of Tyr142 phosphorylation, the kinetics of the phosphorylation/dephosphorylation cycle of $\gamma$-H2AX may be altered. Following this finding, the phosphatase EYA (eye absent) responsible for dephosphorylating H2AX at Tyr142 was identified [21]. Dephosphorylation of Tyr142 was suggested to be a prerequisite for $\gamma \mathrm{H} 2 \mathrm{AX}$ to be recognized by damage repair proteins. When persistent phosphorylation at Tyr142 happens during DNA damage, MDC1-dependent binding of DNA repair factors is inhibited, but recruitment of pro-apoptotic factors, such as JNK1, is promoted [21]. A more recent study demonstrated that the doubly phosphorylated H2AX interact with Microcephalin (MCPH1), an early DNA damage response protein [22]. Although the exact functions of such interaction is still unknown, we speculate that the precise regulation of $\gamma \mathrm{H} 2 \mathrm{AX}$ will be an area of great potential for future DNA damage studies.

\subsection{Poly(ADP-ribosyl)ation}

In addition to $\mathrm{H} 2 \mathrm{AX}$-dependent recruitment, several additional pathways have also been shown to direct the recruitment of various proteins to DNA lesion. Poly(ADP-ribosyl)ation (PARylation) is one of the early events in DDR [2]. Poly(ADP-ribose) polymerases-1 (PARP-1), the founding member of PARP family, sense DNA break through its zinc-finger domain. Structural studies also showed that engaging into the damaged DNA causes PARP-1 conformation change and increases the dynamics of its catalytic domain [23]. In this way, the occurrence of a DNA break is immediately translated into a posttranslational modification of histones $\mathrm{H} 1$ and H2B leading to chromatin structure change [24]. Two waves of accumulation of PARP-1 were observed in living cells. The initial recruitment of PARP-1 activates and locates poly(ADP-ribose) synthesis, which in turn generates binding sites for a second wave of PARP-1 recruitment and other DDR proteins [25]. Recently, it was found that polycomb group (PcG) members and nucleosome remodeling and deacetylase (NuRD) complex are recruited by PARP-1 and -2 to DNA lesions [26]. Both PcG and NuRD are negative regulators of gene transcription, and indeed, rapid loss of nascent RNA and elongating RNA polymerase were 
observed at DNA damage sites. This finding suggests that part of PARP's regulatory role in DDR involves repression of transcription.

\subsection{Sumoylation}

Sumoylation, the covalent attachment of the small proteins known as SUMO (small ubiquitin modifier) to protein substrate, is a very dynamic and reversible PTM [27]. Compared to ubiquitination, knowledge about sumoylation in DDR is relative rudimentary. In 2009, two papers demonstrated the importance of this ubiquitin-like protein modification in DDR [28, 29]. A series of immunofluorescence and live-cell image experiments showed that components in the sumoylation pathway, including enzymes E1 (SAE1), E2 (Ubc9), two of the diverse E3 enzymes (PIAS1 and PIAS4) and the conjugates SUMO1, 2 and 3, are rapidly recruited to DNA damage sites. Functionally, sumoylation of BRCA1 is necessary for its ubiquitin ligase activity. While association of 53BP1, BRCA1 and RNF168 with the DNA damage sites requires accumulation of PIAS1 and PIAS4 to the damaged sites [29].

Several more studies have further revealed that sumoylation and ubiquitination signaling pathways are integrated in the cellular response to DNA damage. For example, two groups showed that the human RNF4, a SUMO-targeted ubiquitin E3 ligase, was recruited to DSBs depending on its SUMO interacting motifs [30,31] Depletion of RNF4 impairs ubiquitin adduct formation at DSB sites, causes persistent histone H2AX phosphorylation [30] and affects the clearance of 53BP1, RNF8, and RNF168 from DNA damage foci [31]. It is proposed that through physical interaction with the SUMO moiety, RNF4 promotes DNA repair by mediating ubiquitylation of sumoylated DDR components at sites of DNA damage.

The role of sumoylation in DNA repair is emphasized by modification of the RPA (replication protein A) complex [32]. RPA was found to physically associate with a SUMO specific protease, SENP6, to maintain its desumoylation status in normal conditions [32]. Under DNA damage, such as those caused by campothecin or IR, the $70 \mathrm{kD}$ subunit of RPA is sumoylated, which in turn recruits Rad51 to DNA lesions, initiating DNA repair through HR. In addition to the specific study of RPA, a recent study in yeast identified a large group of proteins participating in DNA repair and undergoing sumoylation. They showed that defective sumoylation results in failure to complete replication of a damaged genome and impaired DNA end processing, highlighting the importance of sumoylation in maintaining genome stability [33].

\section{DNA damage processed in heterochromatin}

\subsection{The heterochromatin feature}

Chromatin can be divided into euchromatin and heterochromatin, on the basis of differential compaction at interphase. Euchromatin is loosely compacted, more accessible to transcriptional machinery and thus usually actively transcribed. Heterochromatin is typically densely packed, and was previously thought to be inaccessible to the transcription components [34]. Molecularly, heterochromatin is featured with specific histone modifications, such as di- or 
tri-methylation of histone $\mathrm{H} 3$ at lysine 9, and the subsequent recruitment of chromatin association protein such as heterochromatin protein1 (Hp1) [35]. Heterochromatin can be further divided into two groups. First is the constitutive heterochromatin, which contains a high density of repetitive DNA elements, such as satellite sequences and transposable elements. They remain condensed throughout the cell cycle. A second group is facultative heterochromatin, which is dynamic chromosomal loci, condensation of which is regulated by cellular and environmental signals [36].

\subsection{The functions of heterochromatin}

The major function of heterochromatin is to repress transcription and recombination of the embedded repetitive DNA sequences. Disruption of heterochromatin increases the occurrence of spontaneous DSBs, leads to the expansion of DNA repeat arrays, and is correlated with chromosomal defects, such as translocations and loss of heterozygosity [37]. Mechanistically, methylated $\mathrm{H} 3$ at Lysine9 (H3K9me) and the chromatin-bound Hp1 serve as a platform, recruiting various proteins to maintain the highly compact feature of heterochromatin. For example, the HDAC Clr3 is recruited to heterochromatic domains by the yeast Hp1 homolog Swi6. Deacytylation by $\mathrm{Clr} 3$ stabilizes $\mathrm{H} 3$ tri-methylation, increase chromatin condensation and precludes access of Polymerase II [38]. In addition to physically preventing the access of transcription machinery, heterochromatin structure also promotes the post-transcriptional silencing of repetitive sequences. This function is achieved by preferentially targeting the RNA interference components, such as RITS (RNA-induced transcriptional gene silencing) and RDRC (RNA-directed RNA polymerase complex) through H3K9me and Hp1 [39] [40, 41]. On the other side, the recruited RNAi machinery can also contribute to the heterochromatic architecture. In mammalian and drosophila cells, Hp1 shows RNA binding activity, which is required for assembling of condensed chromatin [42, 43]. It is proposed that the RNA derived from repetitive DNA sequence might function as a glue to promote folding or clustering of dispersed heterochromatic loci $[36,41]$.

\subsection{Detection of DSBs in heterochromatin: focusing on $\gamma \mathrm{H} 2 \mathrm{~A}$ foci}

With the realization of heterochromatin structure and functions, the question is how DNA damages in heterochromatin are detected and repaired. In the following sections, we will discuss the recent understanding about these issues.

Abundant reports suggest that heterochromatin is refractory to $\gamma \mathrm{H} 2 \mathrm{~A}$ foci formation upon ionizing radiation [44-46]. However, it remains to be determined whether this phenomenon is due to inaccessible to phosphorylation of $\mathrm{H} 2 \mathrm{AX}$, or heterochromatin is more resistant to DNA damage. Particularly, following DNA damage, chromatin in the vicinity of damaged sites are rapidly de-condensed, which makes the idea that $\gamma \mathrm{H} 2 \mathrm{~A}$ foci is absent in highly packed chromatin a topic of debate [46-48]. On the other side, a recent study utilizing fluorescence in situ hybridization found that the high amount of proteins bound to heterochromatin, including $\mathrm{Hp} 1$, acts as a protective layer that prevents access to the DNA. Therefore, it seems that heterochromatin may internally act as an isolator to inhibit DNA damage [49]. 
However, those thoughts were revisited by a recent study conducted in drosophila cells. In 2011, Chiolo et al. demonstrated that $\gamma$ H2A foci can be formed in heterochromatin upon DSBs [50]. Through a serious of live-cell images and immunofluorescence studies, they found that DSBs and $\gamma \mathrm{H} 2 \mathrm{~A}$ foci were absent at later time points of IR-induced DSBs ( $>60$ mins post IR), which is consistent with previous studies. However, at earlier time points of IR treatment $(<10$ min), both $\gamma \mathrm{H} 2 \mathrm{Ax}$ and ATRIP foci can be observed in heterochromatin, with a level equal to that of non-heterochromatic sites. This study suggests that a complete DDR can occur within heterochromatin (Figure 2A).

\subsection{DSBs repair in heterochromatin}

The next question is how cell repairs the DSBs in heterochromatin. Two issues are raised when considering repair of DNA lesions in heterochromatin. The first is that chromatin compaction in heterochromatin might restrict the access of DDR proteins to damaged sites. Indeed, it was found that DSB repair occurs with slower kinetics and is less effective in heterochromatin [51]. Furthermore, a delay in repair of heterochromatic DSBs was observed in human cells [52]. To overcome the challenge given by tightly compacted chromatin, it was found that the cell can employ the ATM signaling pathway to relax chromatin [51]. Goodarzi et al. found that ATM signaling was specifically required for DSBs repair within heterochromatin, by phosphorylating a transcription repressor, KAP1 (KRAB-associated protein1). KAP1 induces transcriptional repression and chromatin condensation through recruitment of Hp1 [53] and Mi2 $\alpha$ [54]. In the absence of ATM, association of KAP1 to chromatin was increased, suggesting phosphorylation by ATM decreases the affinity of KAP1 for chromatin, which in turn reduces chromatin condensation [51].

The second issue regarding repair of DSBs in heterochromatin is whether NHEJ or HR pathway occurs. In the presence of the closely clustered repeats, HR might produce dicentric and acentric chromosomes, which are known to contribute to human diseases such as cancer and infertility [55]. In this sense, it would be very risky for cells to choose HR to repair the DSBs, since this may lead to abnormal genome rearrangements. In other words, NHEJ repair seems less problematic because small deletions or mutations generally do not affect the function of tandem repeats as severely as genes. However, reports form Chiolo et al. demonstrated that DSBs occuring in heterochromatin are repaired by HR, but the underlying mechanism is distinct from euchromatin [50] (Figure 2A). The most prominent difference is the exclusion of Rad51, which mediates strand invasion, from the DSBs in the heterochromatic domain. Exclusion of Rad51 is achieved by protrusion of heterochromatin, which facilitates DSBs relocalization to the Hp1 $\alpha$ periphery. The movement of the DSBs from inside to outside of heterochromatin depends on checkpoint proteins, such as ATR and resection proteins. Furthermore, relocalization of heterochromatic DSBs is blocked by the Smc5/6 SUMO ligase complex, the yeast homolog of which is required to prevent recombinational repair within the repetitive rDNA locus [56]. It is proposed that Smc5/6 could catalyze sumoylation of one or more components of the recombination machinery and block further assembly of the HR machinery [57]. 
Together, multiple mechanisms exist to guarantee proper DDR in heterochromatin to repair the damaged DNA without compromising genome stability. With improvements in live-cell imaging, we speculate that more details, like the process of DNA damage-induced heterochromatin expansion and reunion of the repaired heterochromatic region, will be revealed.

\section{Human aging and chromatin remodeling}

Aging is a complex process that has been long thought to be a consequence of unprogrammed deleterious events and accumulation of random gene mutation. However, with extensive studies in yeast, worm and mouse, and with the research in premature human aging disease, novel insights have been gained into the molecular mechanisms underlying aging. In this section, we will focus on recent understanding about the contributions that chromatin defects and DNA damage have on human aging.

\subsection{Heterochromatin defects in human aging}

Through studying the premature aging disorder Hutchinson-Gilford Progeria Syndrome (HGPS), the molecular mechanisms leading to chromatin defects in aging are being uncovered. HGPS is an extremely rare genetic disease caused by a point mutation in the LMNA gene, which encodes the major structural protein Lamin A in the nuclear envelope [58]. LMNA mutation leads to abnormal splicing defects and consequent production of a truncated form of lamin A protein, referred to as progerin [59,60]. Notably, in healthy individuals, the same splice site in lamin A was used to cause age-related nuclear defects [61], suggesting conserved mechanism might be shared by both premature and physical human aging process.

One hallmark of human aging, and also in the aging process of other species, is global change in chromatin structure [62, 63].Particularly, loss of heterochromatin structure, loss of heterochromatin proteins and altered patterns of histone modifications, such as decreased H3K9me3, are found in both physiological and premature aging [61, 64-67]. Furthermore, more open chromatin structure, as indicated by tri-methylation at histone 3 lysine 4 , is implicated in shorter lifespan in worm $[68,69]$.

\subsection{Molecular mechanisms underlying heterochromatin loss and aging}

The above evidence suggests that heterochromatin maintenance is critical for longevity. Now the question is how such densely compacted chromatin structure regulates human aging. Unfortunately, due to the complex nature of the aging process and hence experimentally intractability, it is hard to find a direct causal-effect relationship. But recent studies do shed light on the heterochromatic sequence transcription and human aging. Shumaker et al found that associated with the down-regulation of $\mathrm{H} 3 \mathrm{~K} 9 \mathrm{me} 3$, the satellite III repeat transcripts, which is locating in the pericentric heterochromatin, were up-regulated. This up-regulation seems to be sequence-specific since transcription of other group of pericentric repeat, such as $\alpha$ satellite, was not altered [66]. Interestingly, Larson et al. reported that during Drosophila aging, loss of heterochromatin leads to an increased transcription of ribosomal DNA [67]. This rDNA locus 
contains exceeded ribosomal genes and usually only $10 \%$ of them are transcribed [70]. How abnormal transcription of heterochromatic sequences regulate aging is currently unknown. But it is proposed that loss of heterochromatic repeat silencing may affect gene expression patterns and hence affect the integrity of the transcriptome. It is also intriguing to link the heterochromatin status, ribosomal RNA synthesis and aging by taking energy metabolism into account. That is, ribosomal RNA transcription is a rate-limiting step in protein synthesis, increased RNA transcription would promote growth and accelerate aging [71, 72]

\subsection{DNA damage and human aging}

Persistent DNA damage is another hallmark in human aging. Most human premature aging syndromes are caused by various types of DNA damage, and in particular, DSBs [73, 74]. There is no doubt that chromatin defects and DNA damage are major contributors to human aging. The question is which one comes first? Do the DNA damage and the DDR lead to chromatin defects and thus human aging? Or it is that loss of chromatin structure makes the cell more susceptible to DNA damage, increases genome instability, and therefore promotes human aging? Observations made by Pegoraro et al. support that chromatin defects occur prior to DNA damage [75]. They identified that NURD, a protein complex involved in establishment of heterochromatin [76], is a key modulator in aging-associated chromatin defects. Knocking down a subunit of NURD complex lead to aberrant chromatin structure (indicated by loss of H3K9me3 foci) about $50 \mathrm{~h}$ earlier than DNA damage (indicated by existence of $\gamma \mathrm{H} 2 \mathrm{AX}$ foci). This observation suggests that epigenetic and chromatin structure changes are in the upstream of DNA damage events.

How could aberrant chromatin structure cause DNA damage and thus human aging? There might be two mechanisms. 1) The heterochromatic sequences are highly repetitive. Loss of chromatin condensation can leads to abnormal recombination of such sequence and thus genome rearrangement; 2) Heterochromatin confirmation can protect DNA from various insults and repair the damaged lesion with specific mechanisms, as mentioned above.

The next question is how DNA damage leads to human aging? Numerous studies showed that DNA damage and mutations accumulate in human aging. In aged human cells, cytogenetically visible lesions such as translocations, insertions, dicentrics, and acentric fragments are frequently detected [77]. Several signaling pathways linking DNA damage and aging are also proposed, such as the ATM-p53 axis [77] and the BRCA1 dependent aging process [78].However, in addition to being a driver in the accumulation of mutation, recent reports imply that DNA damage-induced RNA transcription change might be a novel mechanism leading human aging. The involvement of RNA processing components in DNA repair strengthens the role of DNA damage in global gene expression changes. For example, a proteomic screen for mediators of DDR showed that enrichment for RNA processing factors, such as splicing-regulator phosphatase PPM1G [79]. Furthermore, the deregulation of gene expression induced by DNA damage resembles increased transcription heterogeneity seen in aged heart tissue [80]. Specifically, Francia et al. found that small RNA produced by DICER and DROSHA, two RNases type III enzymes that process non-coding RNA [81], are required to activate DDR and efficiently repair DNA at the damaged sites [82]. The DICER- and 
DROSHA-dependent small RNA products have the sequence of the damaged locus, and can restore DDR in RNase-treated cells [82]. Intriguingly, these DNA damage-induced RNA can regulate cellular senescence in cultured human and mouse cells, and in living zebrafish larvae [82]. Genetic ablation of DICER has been reported to cause premature senescence in both developing and adult mice [83]. Do the DICER- and DROSHA-dependent, DNA damagedinduced RNAs affect heterochromatin structure and thus regulating cell senescence and human aging? Although the methylation status of H3K9 did not alter with DICER or DROSHA knockdown [82], it is still interesting to check if other heterochromatic proteins, such as Hp1, which has RNA binding activity, could be affected (Figure 2). Furthermore, does the sitespecific RNA production have any role in the dynamic movement of heterochromatic domain during DNA damage, for example, the protrusion of heterochromatin? Answering these questions will be important to gain a comprehensive understanding of the interplay between chromatin structure and RNA transcription during DDR.

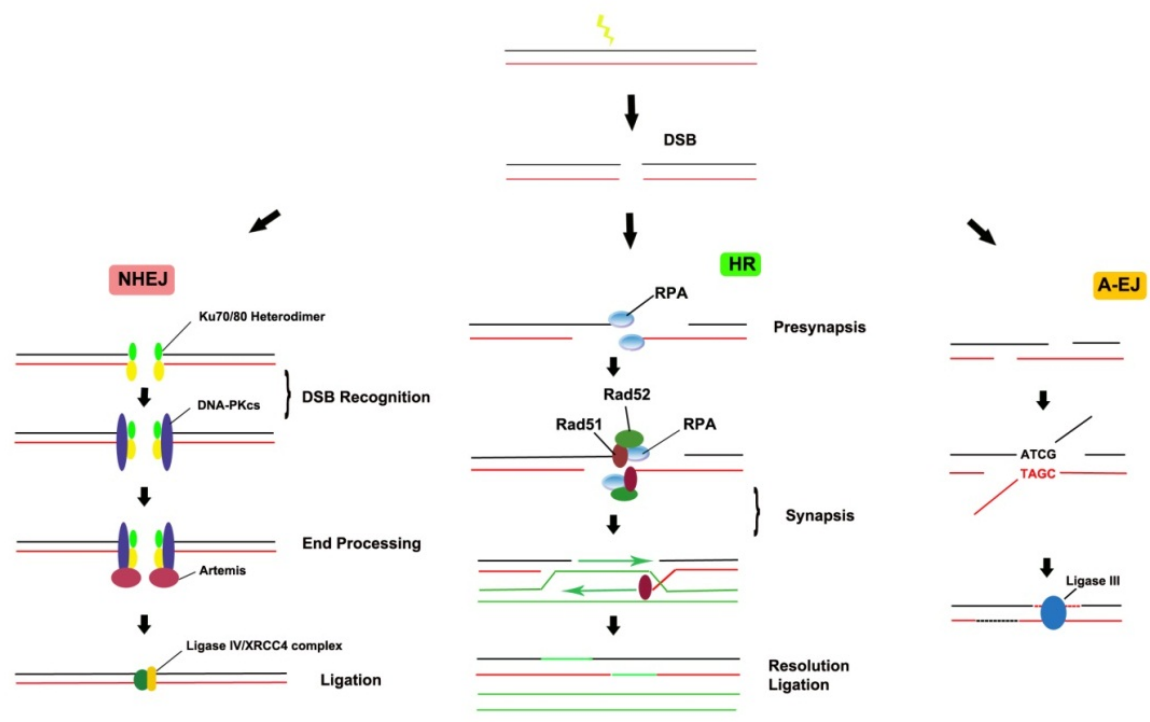

Figure 1. In NHEJ, the heterodimer Ku70/80 interacts with the end of damaged DNA and recruits DNA-PKcs. Artemis, which processes the ends of DNA and makes them compatible for ligation, is also recruited. Finally, the DNA breaks were joined by XRCC4/Ligase IV. In HR, a homologous stretch on a sister chromatid is utilized to accurately repair the DSB. DNA ends are first processed in order to create single strand overhangs. RPA is then coated to these overhangs, which recruits Rad51 and other co-factors such as Rad52. The Rad51 coated DNA filament then invade the undamaged strands, and a joint molecule is formed by the damaged and undamaged strands. Finally, template guides DNA synthesis and resolution of the two strands. A-EJ shares the initial resection step with HR but it requires neither extended resection nor extended sequence homology. DNA ends that are not bound by Ku70/80 are degraded. Single strand DNA resection reveals 2-4 (indicated by ATCG in the figure) or more nucleotides which can anneal, creating branched intermediate structures. The resolution of this intermediate structure results in deletions at the repair junctions. A-EJ is independent of Ku70/80 but dependent on Ligase III to join the DNA ends. 


\section{Conclusions}

Since discovery of the function of $\gamma \mathrm{H} 2 \mathrm{AX}$ in DDR, dynamic regulation of chromatin in response to DNA damage has received great attention during these past decades. In this chapter, we have discussed the contribution of three posttranslational modifications: phosphorylation, PPAR and sumoylation in DDR. We also discussed the current understanding about heterochromatin changes during DDR and how it regulates human aging. We emphasize the function of heterochromatin in DDR because this condensed chromatin structure is particularly involved in human aging. Emerging evidence suggests that heterochromatin is not refractory to DNA damage, but utilize a different downstream mechanism to repair the damaged sites and thus prevent unwanted genome recombination. DNA damage may change the heterochromatin structure by abnormal generation of non-coding RNA at the damaged locus, which could titrate the RNA-binding heterochromatin proteins (such as Hp1) and lead to subsequent abnormal heterochromatin structure. The integrity of the transcriptome can also be affected by DNA damage and regulate human aging. We speculate that the pathways regarding DNA damage repair in heterochromatin and the interplay between heterochromatic sequence transcription and human aging will be a hot area for future research (Figure 3).

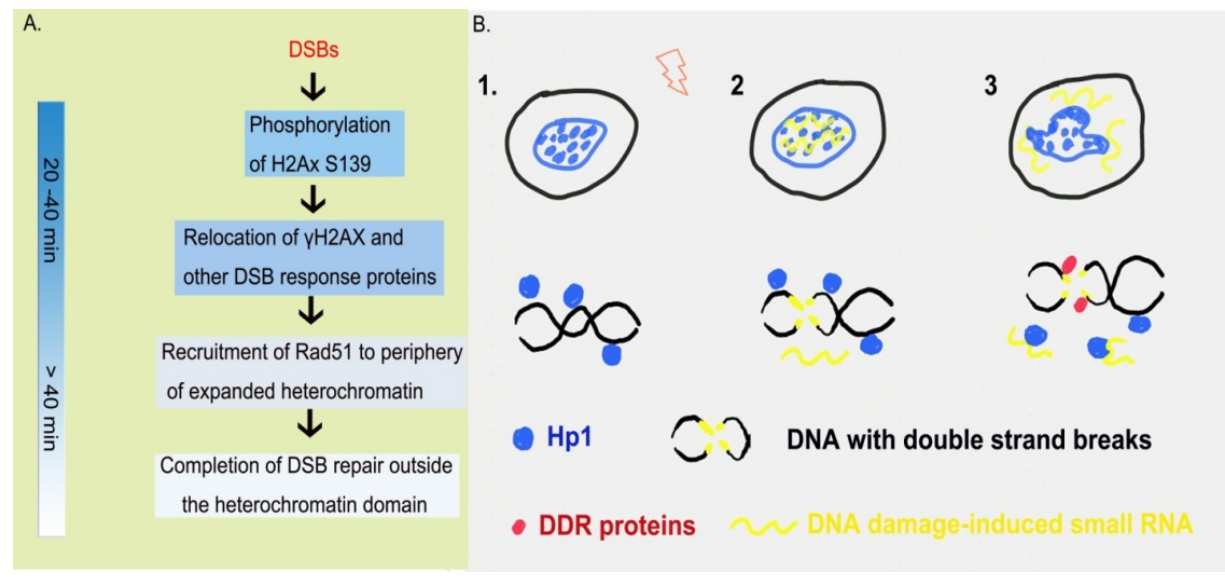

Figure 2. A. Schematic diagram shows DNA damage response in heterochromatin. Initial DSB recognition is very rapid in heterochromatin. Rapid phosphorylation and relocalization of H2Ax S139 and other DSB proteins (such as ATRIP) occur in heterochromatic region. Rad51 recombinase recruitment is inhibited in Hp1-rich heterochromatin, which allows DSB processing to induce heterochromatin expansion and DSB repaired in euchromatic site. In this way, unwanted homologous recombination is prevented in heterochromatin. B. A potential model for DNA damage response in heterochromatin. A typical heterochromatic domain is indicated by the enrichment of Hp1 protein (step 1). Under DNA damage, double strand breaks occur, which might result in production of site-specific small RNA with sequence of the damaged site (step 2). Hp1 might bind to the DNA damage-induced small RNA, which leads to its relocalization and consequent dynamic movement of the heterochromatic domain during DNA damage. Binding of Hp1 to the small RNA might also facilitate recruiting of other DDR proteins to the damaged heterochromatic sequences, and thus promoting damage repair. Notably, it still needs to be determined whether the damage-induced RNA is generated in heterochromatin (step [2]). If this is the case, the role of this RNA in the dynamic movement of heterochromatin during DNA damage also awaits investigation (step 3). 

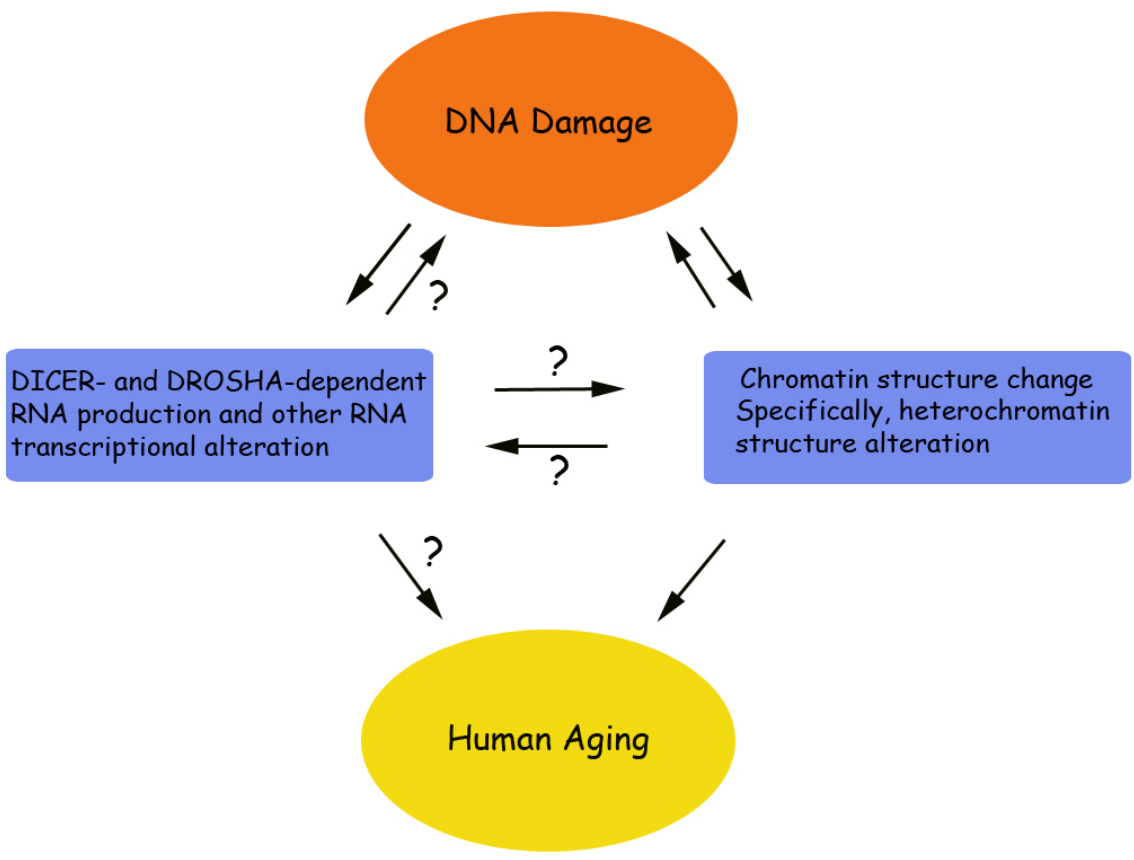

Figure 3. Interplay between DNA damage, chromatin remodeling and human aging. It is well recognized that DNA damage leads to chromatin remodeling. On the other hand, abnormal chromatin structures also contribute to DNA damage and correlate with human aging. Emerging evidence shed light on the DNA damage-induced global transcription regulation and also locus-specific production of small RNAs. It would be interesting to know through which mechanism the DNA damage-induced RNA regulates DNA damage response, and whether it affects the chromatin, especially, heterochromatin structures, and hence modulate human aging.

\section{Author details}

Lili Gong, Edward Wang and Shiaw-Yih Lin

Department of Systems Biology, The University of Texas M. D. Anderson Cancer Center, Houston, Texas, USA

\section{References}

[1] Kornberg RD. Chromatin structure: a repeating unit of histones and DNA. Science. 1974 May 24;184(4139):868-71. 
[2] Polo SE, Jackson SP. Dynamics of DNA damage response proteins at DNA breaks: a focus on protein modifications. Genes Dev. 2011 Mar 1;25(5):409-33.

[3] Lukas J, Lukas C, Bartek J. More than just a focus: The chromatin response to DNA damage and its role in genome integrity maintenance. Nat Cell Biol. 2011 Oct;13(10): 1161-9.

[4] Gellert M. V(D)J recombination: RAG proteins, repair factors, and regulation. Annu Rev Biochem. 2002;71:101-32.

[5] Jackson SP, Bartek J. The DNA-damage response in human biology and disease. Nature. 2009 Oct 22;461(7267):1071-8.

[6] Friedberg EC. Nucleotide excision repair of DNA: The very early history. DNA Repair. 2011 Jul 15;10(7):668-72.

[7] Symington LS, Gautier J. Double-strand break end resection and repair pathway choice. Annu Rev Genet. 2011;45:247-71.

[8] Lieber MR. The mechanism of double-strand DNA break repair by the nonhomologous DNA end-joining pathway. Annu Rev Biochem. 2010;79:181-211.

[9] Weterings E, Chen DJ. The endless tale of non-homologous end-joining. Cell Res. 2008 Jan;18(1):114-24.

[10] Jeggo PA. DNA breakage and repair. Adv Genet. 1998;38:185-218.

[11] Haber JE. Partners and pathwaysrepairing a double-strand break. Trends Genet. 2000 Jun;16(6):259-64.

[12] Baumann P, West SC. Role of the human RAD51 protein in homologous recombination and double-stranded-break repair. Trends Biochem Sci. 1998 Jul;23(7):247-51.

[13] Gasior SL, Wong AK, Kora Y, Shinohara A, Bishop DK. Rad52 associates with RPA and functions with rad55 and rad57 to assemble meiotic recombination complexes. Genes Dev. 1998 Jul 15;12(14):2208-21.

[14] Sugawara N, Wang X, Haber JE. In vivo roles of Rad52, Rad54, and Rad55 proteins in Rad51-mediated recombination. Mol Cell. 2003 Jul;12(1):209-19.

[15] Rass E, Grabarz A, Plo I, Gautier J, Bertrand P, Lopez BS. Role of Mre11 in chromosomal nonhomologous end joining in mammalian cells. Nat Struct Mol Biol. 2009 Aug; 16(8):819-24.

[16] Rogakou EP, Pilch DR, Orr AH, Ivanova VS, Bonner WM. DNA double-stranded breaks induce histone H2AX phosphorylation on serine 139. J Biol Chem. 1998 Mar 6;273(10):5858-68.

[17] O'Hagan HM, Mohammad HP, Baylin SB. Double strand breaks can initiate gene silencing and SIRT1-dependent onset of DNA methylation in an exogenous promoter CpG island. PLoS Genet. 2008;4(8):e1000155. 
[18] Cuozzo C, Porcellini A, Angrisano T, Morano A, Lee B, Di Pardo A, et al. DNA damage, homology-directed repair, and DNA methylation. PLoS Genet. 2007 Jul; 3(7):e110.

[19] Harper JW, Elledge SJ. The DNA damage response: ten years after. Mol Cell. 2007 Dec 14;28(5):739-45.

[20] Xiao A, Li H, Shechter D, Ahn SH, Fabrizio LA, Erdjument-Bromage H, et al. WSTF regulates the H2A.X DNA damage response via a novel tyrosine kinase activity. Nature. 2009 Jan 1;457(7225):57-62.

[21] Cook PJ, Ju BG, Telese F, Wang X, Glass CK, Rosenfeld MG. Tyrosine dephosphorylation of $\mathrm{H} 2 \mathrm{AX}$ modulates apoptosis and survival decisions. Nature. $2009 \mathrm{Apr}$ 2;458(7238):591-6.

[22] Singh N, Basnet H, Wiltshire TD, Mohammad DH, Thompson JR, Heroux A, et al. Dual recognition of phosphoserine and phosphotyrosine in histone variant H2A.X by DNA damage response protein MCPH1. Proc Natl Acad Sci U S A. 2012 Sep 4;109(36):14381-6.

[23] Langelier MF, Planck JL, Roy S, Pascal JM. Structural basis for DNA damage-dependent poly(ADP-ribosyl)ation by human PARP-1. Science. 2012 May 11;336(6082): 728-32.

[24] Messner S, Altmeyer M, Zhao H, Pozivil A, Roschitzki B, Gehrig P, et al. PARP1 ADP-ribosylates lysine residues of the core histone tails. Nucleic Acids Res. 2010 Oct; 38(19):6350-62.

[25] Mortusewicz O, Ame JC, Schreiber V, Leonhardt H. Feedback-regulated poly(ADPribosyl)ation by PARP-1 is required for rapid response to DNA damage in living cells. Nucleic Acids Res. 2007;35(22):7665-75.

[26] Chou DM, Adamson B, Dephoure NE, Tan X, Nottke AC, Hurov KE, et al. A chromatin localization screen reveals poly (ADP ribose)-regulated recruitment of the repressive polycomb and NuRD complexes to sites of DNA damage. Proc Natl Acad Sci U S A. 2010 Oct 26;107(43):18475-80.

[27] Wilkinson KA, Henley JM. Mechanisms, regulation and consequences of protein SUMOylation. Biochem J. 2010 Jun 1;428(2):133-45.

[28] Galanty Y, Belotserkovskaya R, Coates J, Polo S, Miller KM, Jackson SP. Mammalian SUMO E3-ligases PIAS1 and PIAS4 promote responses to DNA double-strand breaks. Nature. 2009 Dec 17;462(7275):935-9.

[29] Morris JR, Boutell C, Keppler M, Densham R, Weekes D, Alamshah A, et al. The SU$\mathrm{MO}$ modification pathway is involved in the BRCA1 response to genotoxic stress. Nature. 2009 Dec 17;462(7275):886-90. 
[30] Galanty Y, Belotserkovskaya R, Coates J, Jackson SP. RNF4, a SUMO-targeted ubiquitin E3 ligase, promotes DNA double-strand break repair. Genes Dev. 2012 Jun 1;26(11):1179-95.

[31] Yin Y, Seifert A, Chua JS, Maure JF, Golebiowski F, Hay RT. SUMO-targeted ubiquitin E3 ligase RNF4 is required for the response of human cells to DNA damage. Genes Dev. 2012 Jun 1;26(11):1196-208.

[32] Dou H, Huang C, Singh M, Carpenter PB, Yeh ET. Regulation of DNA repair through deSUMOylation and SUMOylation of replication protein A complex. Mol Cell. 2010 Aug 13;39(3):333-45.

[33] Cremona CA, Sarangi P, Yang Y, Hang LE, Rahman S, Zhao X. Extensive DNA damage-induced sumoylation contributes to replication and repair and acts in addition to the mec1 checkpoint. Mol Cell. 2012 Feb 10;45(3):422-32.

[34] Huisinga KL, Brower-Toland B, Elgin SC. The contradictory definitions of heterochromatin: transcription and silencing. Chromosoma. 2006 Apr;115(2):110-22.

[35] Cheutin T, McNairn AJ, Jenuwein T, Gilbert DM, Singh PB, Misteli T. Maintenance of stable heterochromatin domains by dynamic HP1 binding. Science. 2003 Jan 31;299(5607):721-5.

[36] Grewal SI, Jia S. Heterochromatin revisited. Nat Rev Genet. 2007 Jan;8(1):35-46.

[37] Peng JC, Karpen GH. Heterochromatic genome stability requires regulators of histone H3 K9 methylation. PLoS Genet. 2009 Mar;5(3):e1000435.

[38] Yamada T, Fischle W, Sugiyama T, Allis CD, Grewal SI. The nucleation and maintenance of heterochromatin by a histone deacetylase in fission yeast. Mol Cell. 2005 Oct 28;20(2):173-85.

[39] Motamedi MR, Verdel A, Colmenares SU, Gerber SA, Gygi SP, Moazed D. Two RNAi complexes, RITS and RDRC, physically interact and localize to noncoding centromeric RNAs. Cell. 2004 Dec 17;119(6):789-802.

[40] Sugiyama T, Cam H, Verdel A, Moazed D, Grewal SI. RNA-dependent RNA polymerase is an essential component of a self-enforcing loop coupling heterochromatin assembly to siRNA production. Proc Natl Acad Sci U S A. 2005 Jan 4;102(1):152-7.

[41] Cam HP, Sugiyama T, Chen ES, Chen X, FitzGerald PC, Grewal SI. Comprehensive analysis of heterochromatin- and RNAi-mediated epigenetic control of the fission yeast genome. Nat Genet. 2005 Aug;37(8):809-19.

[42] Muchardt C, Guilleme M, Seeler JS, Trouche D, Dejean A, Yaniv M. Coordinated methyl and RNA binding is required for heterochromatin localization of mammalian HP1alpha. EMBO Rep. 2002 Oct;3(10):975-81. 
[43] Keller C, Adaixo R, Stunnenberg R, Woolcock KJ, Hiller S, Buhler M. HP1(Swi6) mediates the recognition and destruction of heterochromatic RNA transcripts. Mol Cell. 2012 Jul 27;47(2):215-27.

[44] Cowell IG, Sunter NJ, Singh PB, Austin CA, Durkacz BW, Tilby MJ. gammaH2AX foci form preferentially in euchromatin after ionising-radiation. PLoS One. 2007;2(10):e1057.

[45] Vasireddy RS, Karagiannis TC, El-Osta A. gamma-radiation-induced gammaH2AX formation occurs preferentially in actively transcribing euchromatic loci. Cell Mol Life Sci. 2010 Jan;67(2):291-4.

[46] Cann KL, Dellaire G. Heterochromatin and the DNA damage response: the need to relax. Biochem Cell Biol. 2011 Feb;89(1):45-60.

[47] Kruhlak MJ, Celeste A, Dellaire G, Fernandez-Capetillo O, Muller WG, McNally JG, et al. Changes in chromatin structure and mobility in living cells at sites of DNA double-strand breaks. J Cell Biol. 2006 Mar 13;172(6):823-34.

[48] Falk M, Lukasova E, Gabrielova B, Ondrej V, Kozubek S. Chromatin dynamics during DSB repair. Biochim Biophys Acta. 2007 Oct;1773(10):1534-45.

[49] Falk M, Lukasova E, Kozubek S. Chromatin structure influences the sensitivity of DNA to gamma-radiation. Biochim Biophys Acta. 2008 Dec;1783(12):2398-414.

[50] Chiolo I, Minoda A, Colmenares SU, Polyzos A, Costes SV, Karpen GH. Doublestrand breaks in heterochromatin move outside of a dynamic HP1a domain to complete recombinational repair. Cell. 2011 Mar 4;144(5):732-44.

[51] Goodarzi AA, Noon AT, Deckbar D, Ziv Y, Shiloh Y, Lobrich M, et al. ATM signaling facilitates repair of DNA double-strand breaks associated with heterochromatin. Mol Cell. 2008 Jul 25;31(2):167-77.

[52] Noon AT, Shibata A, Rief N, Lobrich M, Stewart GS, Jeggo PA, et al. 53BP1-dependent robust localized KAP-1 phosphorylation is essential for heterochromatic DNA double-strand break repair. Nat Cell Biol. 2010 Feb;12(2):177-84.

[53] Lechner MS, Begg GE, Speicher DW, Rauscher FJ, 3rd. Molecular determinants for targeting heterochromatin protein 1-mediated gene silencing: direct chromoshadow domain-KAP-1 corepressor interaction is essential. Mol Cell Biol. 2000 Sep;20(17): 6449-65.

[54] Schultz DC, Friedman JR, Rauscher FJ, 3rd. Targeting histone deacetylase complexes via KRAB-zinc finger proteins: the PHD and bromodomains of KAP-1 form a cooperative unit that recruits a novel isoform of the Mi-2alpha subunit of NuRD. Genes Dev. 2001 Feb 15;15(4):428-43.

[55] Pearson CE, Nichol Edamura K, Cleary JD. Repeat instability: mechanisms of dynamic mutations. Nat Rev Genet. 2005 Oct;6(10):729-42. 
[56] Torres-Rosell J, Sunjevaric I, De Piccoli G, Sacher M, Eckert-Boulet N, Reid R, et al. The Smc5-Smc6 complex and SUMO modification of Rad52 regulates recombinational repair at the ribosomal gene locus. Nat Cell Biol. 2007 Aug;9(8):923-31.

[57] Peterson CL. The ins and outs of heterochromatic DNA repair. Dev Cell. 2011 Mar $15 ; 20(3): 285-7$.

[58] Kudlow BA, Kennedy BK, Monnat RJ, Jr. Werner and Hutchinson-Gilford progeria syndromes: mechanistic basis of human progeroid diseases. Nat Rev Mol Cell Biol. 2007 May;8(5):394-404.

[59] De Sandre-Giovannoli A, Bernard R, Cau P, Navarro C, Amiel J, Boccaccio I, et al. Lamin a truncation in Hutchinson-Gilford progeria. Science. 2003 Jun 27;300(5628): 2055.

[60] Eriksson M, Brown WT, Gordon LB, Glynn MW, Singer J, Scott L, et al. Recurrent de novo point mutations in lamin A cause Hutchinson-Gilford progeria syndrome. Nature. 2003 May 15;423(6937):293-8.

[61] Scaffidi P, Misteli T. Lamin A-dependent nuclear defects in human aging. Science. (Research Support, N.I.H., Intramural). 2006 May 19;312(5776):1059-63.

[62] Pegoraro G, Misteli T. The central role of chromatin maintenance in aging. Aging (Albany NY). 2009 Dec;1(12):1017-22.

[63] Swedlow J, Danuser G. Scale integration: the structure and dynamics of macromolecular assemblies, cells and tissues. Curr Opin Cell Biol. 2012 Feb;24(1):1-3.

[64] Goldman RD, Shumaker DK, Erdos MR, Eriksson M, Goldman AE, Gordon LB, et al. Accumulation of mutant lamin A causes progressive changes in nuclear architecture in Hutchinson-Gilford progeria syndrome. Proc Natl Acad Sci U S A. 2004 Jun 15;101(24):8963-8.

[65] Scaffidi P, Misteli T. Reversal of the cellular phenotype in the premature aging disease Hutchinson-Gilford progeria syndrome. Nat Med. 2005 Apr;11(4):440-5.

[66] Shumaker DK, Dechat T, Kohlmaier A, Adam SA, Bozovsky MR, Erdos MR, et al. Mutant nuclear lamin A leads to progressive alterations of epigenetic control in premature aging. Proc Natl Acad Sci U S A. 2006 Jun 6;103(23):8703-8.

[67] Larson K, Yan SJ, Tsurumi A, Liu J, Zhou J, Gaur K, et al. Heterochromatin formation promotes longevity and represses ribosomal RNA synthesis. PLoS Genet. 2012 Jan; 8(1):e1002473.

[68] Hamilton B, Dong Y, Shindo M, Liu W, Odell I, Ruvkun G, et al. A systematic RNAi screen for longevity genes in C. elegans. Genes Dev. 2005 Jul 1;19(13):1544-55.

[69] Han S, Brunet A. Histone methylation makes its mark on longevity. Trends Cell Biol. 2012 Jan;22(1):42-9. 
[70] Eickbush DG, Ye J, Zhang X, Burke WD, Eickbush TH. Epigenetic regulation of retrotransposons within the nucleolus of Drosophila. Mol Cell Biol. 2008 Oct;28(20): 6452-61.

[71] Hansen M, Taubert S, Crawford D, Libina N, Lee SJ, Kenyon C. Lifespan extension by conditions that inhibit translation in Caenorhabditis elegans. Aging Cell. 2007 Feb;6(1):95-110.

[72] Pan KZ, Palter JE, Rogers AN, Olsen A, Chen D, Lithgow GJ, et al. Inhibition of mRNA translation extends lifespan in Caenorhabditis elegans. Aging Cell. 2007 Feb; 6(1):111-9.

[73] Sedelnikova OA, Horikawa I, Redon C, Nakamura A, Zimonjic DB, Popescu NC, et al. Delayed kinetics of DNA double-strand break processing in normal and pathological aging. Aging Cell. 2008 Jan;7(1):89-100.

[74] Sedelnikova OA, Horikawa I, Zimonjic DB, Popescu NC, Bonner WM, Barrett JC. Senescing human cells and ageing mice accumulate DNA lesions with unrepairable double-strand breaks. Nat Cell Biol. 2004 Feb;6(2):168-70.

[75] Pegoraro G, Kubben N, Wickert U, Gohler H, Hoffmann K, Misteli T. Ageing-related chromatin defects through loss of the NURD complex. Nat Cell Biol. 2009 Oct;11(10): 1261-7.

[76] Zhang Y, Ng HH, Erdjument-Bromage H, Tempst P, Bird A, Reinberg D. Analysis of the NuRD subunits reveals a histone deacetylase core complex and a connection with DNA methylation. Genes Dev. 1999 Aug 1;13(15):1924-35.

[77] Lombard DB, Chua KF, Mostoslavsky R, Franco S, Gostissa M, Alt FW. DNA repair, genome stability, and aging. Cell. 2005 Feb 25;120(4):497-512.

[78] Cao L, Li W, Kim S, Brodie SG, Deng CX. Senescence, aging, and malignant transformation mediated by p53 in mice lacking the Brca1 full-length isoform. Genes Dev. 2003 Jan 15;17(2):201-13.

[79] Beli P, Lukashchuk N, Wagner SA, Weinert BT, Olsen JV, Baskcomb L, et al. Proteomic investigations reveal a role for RNA processing factor THRAP3 in the DNA damage response. Mol Cell. 2012 Apr 27;46(2):212-25.

[80] Bahar R, Hartmann CH, Rodriguez KA, Denny AD, Busuttil RA, Dolle ME, et al. Increased cell-to-cell variation in gene expression in ageing mouse heart. Nature. 2006 Jun 22;441(7096):1011-4.

[81] Kim VN, Han J, Siomi MC. Biogenesis of small RNAs in animals. Nat Rev Mol Cell Biol. 2009 Feb;10(2):126-39.

[82] Francia S, Michelini F, Saxena A, Tang D, de Hoon M, Anelli V, et al. Site-specific DICER and DROSHA RNA products control the DNA-damage response. Nature. 2012 Aug 9;488(7410):231-5. 
[83] Mudhasani R, Zhu Z, Hutvagner G, Eischen CM, Lyle S, Hall LL, et al. Loss of miRNA biogenesis induces p19Arf-p53 signaling and senescence in primary cells. J Cell Biol. 2008 Jun 30;181(7):1055-63. 
